\title{
Molecular and Gene Network Analysis of Thyroid Transcription Factor 1 (TTF1) and Enhanced at Puberty (EAP1) Genes in Patients with GnRH-Dependent Pubertal Disorders
}

\author{
Priscilla Cukier $^{a}$ Hollis Wright ${ }^{b}$ Tomke Rulfs ${ }^{c}$ Leticia Ferreira Gontijo Silveira ${ }^{a}$ \\ Milena Gurgel Teles ${ }^{a}$ Berenice Bilharinho Mendonca ${ }^{a}$ Ivo J.P. Arnhold ${ }^{a}$ \\ Sabine Heger ${ }^{c}$ Ana Claudia Latronico ${ }^{a}$ Sergio R. Ojeda ${ }^{b}$ Vinicius Nahime Brito ${ }^{a}$ \\ a Unidade de Endocrinologia do Desenvolvimento, Disciplina de Endocrinologia da Faculdade de Medicina \\ da Universidade de São Paulo e Laboratório de Hormônios e Genética Molecular LIM/42, São Paulo, Brazil; \\ ${ }^{b}$ Division of Neuroscience, Oregon National Primate Research Center/Oregon Health and Science University \\ (ONPRC/OHSU), Beaverton, Oreg., USA; ' Children's Hospital 'Auf der Bult', Institute of Clinical Biochemistry, \\ Hannover Medical School, Hannover, Germany
}

\section{Key Words}

Central precocious puberty $\cdot$ Hypogonadotropic hypogonadism $\cdot$ Hypothalamic hamartoma $\cdot$ TTF1 EAP1

\begin{abstract}
Background/Aim: TTF1 and EAP1 are transcription factors that modulate gonadotropin-releasing hormone expression. We investigated the contribution of TTF1 and EAP1 genes to central pubertal disorders. Patients and Methods: 133 patients with central pubertal disorders were studied: 86 with central precocious puberty and 47 with normosmic isolated hypogonadotropic hypogonadism. The coding region of TTF1 and EAP1 were sequenced. Variations of polyglutamine and polyalanine repeats in EAP1 were analyzed by GeneScan software. Association of TTF1 and EAP1 to genes implicated in timing of puberty was investigated by metanetwork framework GeneMANIA and Cytoscape software. Results: Direct sequencing of the TTF1 did not reveal any mutation or polymorphisms. Four EAP1 synonymous variants were identified with similar frequencies among groups.
\end{abstract}

The most common EAP1 5 '-distal polyalanine genotype was the homozygous $12 / 12$, but the genotype $12 / 9$ was identified in 2 central precocious puberty sisters without functional alteration in EAP1 transcriptional activity. TTF1 and EAP1 were connected, via genetic networks, to genes implicated in the control of menarche. Conclusion: No TTF1 or EAP1 germline mutations were associated with central pubertal disorders. TTF1 and EAP1 may affect puberty by changing expression in response to other members of puberty-associated gene networks, or by differentially affecting the expression of gene components of these networks.

(c) 2013 S. Karger AG, Basel

\section{Introduction}

Gonadotropin-releasing hormone ( $\mathrm{GnRH})$ secretion from hypothalamic neurosecretory neurons is controlled by transsynaptic inputs of both excitatory and inhibitory nature, as well as by facilitatory glial influences [1]. A coordinated increase in $\mathrm{GnRH}$ secretory activity is crucial

\section{KARGER}

E-Mail karger@karger.com www.karger.com/hrp
(C) 2013 S. Karger AG, Basel

$1663-2818 / 13 / 0804-0257 \$ 38.00 / 0$
Vinicius Nahime Brito

Hospital das Clínicas, Faculdade de Medicina da Universidade de São Paulo Disciplina de Endocrinologia e Metabologia

Av. Dr. Eneas de Carvalho Aguiar, $1552^{\circ}$ andar Bloco 6, São Paulo, SP 05403-900 (Brazil) E-Mail vinicius.brito@hc.fm.usp.br 
for the normal initiation and progression of the pubertal process [1-3]. Thyroid transcription factor-1 (TTF1; also known as NKX2-1), a homeobox gene expressed in the thyroid, lung and brain, and enhanced at puberty (EAP1; also known as IRF2BPL, interferon regulatory factor 2 binding protein-like), a gene of widespread distribution, have been postulated to occupy central positions within gene networks involved in the hypothalamic control of $\mathrm{GnRH}$ production [4-7]. Both encode proteins able to trans-activate the GnRH gene directly and to regulate the expression of genes involved in the stimulatory and inhibitory control of the GnRH neuronal network $[8,9]$. Because of their transcriptional activity and connectivity with other transcriptional regulators, they have been postulated to control the expression of many genes that are necessary for the neuron-to-neuron and glia-to-neuron regulation of $\mathrm{GnRH}$ secretion at puberty [7]. In addition, TTF1 has a crucial role in diencephalic morphogenesis [4, $10]$.

TTF1 and EAP1 expression increases in the mediobasal hypothalamus of both non-human primates and rodents at either the time of puberty or preceding the onset of the pubertal process $[8,9]$. In both cases, TTF1 and/or EAP1 loss-of-function results in delayed pubertal development. For instance, conditional deletion of the Ttf1 gene from neurons resulted in delayed puberty, disruption of initial estrous cyclicity, and decreased reproductive ability in mice [10]. A similar phenotype is observed following EAP1 silencing via RNAi in rodents and nonhuman primates, suggesting that both genes are important for the neuroendocrine control of reproductive development $[9,11]$.

In humans, GnRH-dependent pubertal disorders comprise a range of pathologies in which $\mathrm{GnRH}$ release is disrupted, leading to a wide spectrum of phenotypes varying from central precocious puberty (CPP) (early $\mathrm{GnRH}$ secretion) to isolated normosmic hypogonadotropic hypogonadism (nIHH, lack of GnRH release) [12]. Around $30 \%$ of nIHH cases have a defined genetic basis [13]. In contrast, the vast majority of CPP cases are considered idiopathic with a reported prevalence of about $25 \%$ of familial CPP [14]. Thus far, rare gain-of-function mutations in KISS1R and KISS1 and infrequent KISS1 polymorphisms with possible protective effect were described in sporadic cases of CPP [15-17]. A subset of CPP is caused by hypothalamic hamartoma $(\mathrm{CPPH})$, the most common structural abnormality associated to CPP [1820]. Because little is known about the molecular basis of hypothalamic hamartomas, we included $\mathrm{CPPH}$ patients in this study.
An emerging concept in the field of polygenic human disorders is that the phenotypic manifestations of such disorders are rarely caused by mutations in the DNA sequence of single genes [21]. Instead, mutations conferring risk appear to affect many functionally connected genes, so that the disease ultimately results from the $\mathrm{cu}-$ mulative risk of a large number of independent loci [21]. It may also be envisioned that polygenic disorders, such as those affecting pubertal development, may result from sequence variation affecting many functionally related genes. While such variations may be inconsequential in isolation, their collective manifestation may lead to changes in gene expression which, in turn, could cause functional alterations in gene networks contributing to the disorder. This concept has never been explored in previous attempts to unveil the genetic underpinnings of human pubertal disorders.

Based on these considerations, the present study was undertaken to (a) determine if sequence alterations of the TTF1 and/or EAP1 genes are an underlying cause of some GnRH-dependent pubertal disorders in humans, and (b) to test in silico (i.e. using computational biology tools) the novel concept that the influence of TTF1 and/or EAP1 on the timing of human puberty may be determined by functional associations with a multiplicity of other genes implicated in the control of human puberty.

\section{Patients and Methods}

\section{Patients}

Written informed consent was obtained from the patients or their parents or guardians according to procedures approved by the institutional research ethics committee of each participating institution. All the patients were recruited from the Unit of Developmental Endocrinology Outpatient Clinic at Hospital das Clínicas da Faculdade de Medicina da Universidade de São Paulo, Brazil.

A total of 133 patients with a diagnosis of GnRH-dependent pubertal disorders (86 CPP and $47 \mathrm{nIHH}$ ) were selected. A control group composed of 53 healthy volunteers from both sexes was also studied.

From 86 patients with CPP, 71 patients (67 girls) had the idiopathic form and 15 (7 girls) had CPPH. 18 idiopathic CPP patients $(25 \%)$ originating from 14 distinct families had a familial history of CPP, defined as more than 1 affected patient in the same family [14]. All CPP patients presented with development of secondary sexual features before 8 years of age in girls and 9 years in boys, increased growth velocity, advanced bone age assessed by Greulich \& Pyle method (evaluated by P.C., V.N.B., and A.C.L.) and pubertal basal LH levels (>0.6 U/l) and/or GnRH-stimulated LH peak assessed by radioimmunoassay $(>15 \mathrm{U} / \mathrm{l}$ for girls and $>25 \mathrm{U} / \mathrm{l}$ for boys) or immunofluorometric assay ( $>6.9 \mathrm{U} / \mathrm{l}$ for girls and $>9.6 \mathrm{U} / \mathrm{l}$ for boys) $[19,20]$. All patients with the idiopathic form had a nor- 
mal central nervous system MRI scan. The diagnosis of hypothalamic hamartoma was confirmed by the finding of an isointense mass at MRI scan, generally located at the tuber cinereum $[18,20]$. Clinical and hormonal features of these patients are summarized in table 1. Patients were treated with depot GnRH analogs (leuprolide acetate $3.75 \mathrm{mg}$ monthly) with adequate clinical and hormonal outcome. In 2 unrelated Brazilian patients with idiopathic CPP, a genetic etiology was previously identified: 1 girl who carried an activating KISS1R mutation and 1 boy harboring a KISS1 gain-offunction mutation $[15,16]$.

47 patients (13 women) had nIHH. This diagnosis was confirmed by prepubertal or low testosterone or estradiol levels for chronological age, diminished or inappropriately normal basal and GnRH-stimulated LH and FSH release, normal baseline and stimulated levels of other anterior pituitary hormones and normal hypothalamic-pituitary imaging [22]. Olfactory tests (Smell Identification Test ${ }^{\mathrm{TM}}$, Sensonics, Philadelphia, Pa., USA, or Alcohol Sniff Test, UCSD Nasal Dysfunction Clinic, San Diego, Calif., USA) ruled out olfactory abnormalities [23]. All patients whose first evaluation was before 16 years of age were followed until adulthood to confirm the diagnosis of IHH. Clinical and hormonal features of these patients are presented in table 1. A familial form of IHH was identified in 11 of 47 patients (23\%). Previous studies from our laboratory identified $11(23 \%) \mathrm{nIHH}$ patients harboring genetic abnormalities in the following genes: FGFR1, FGF8, GNRHR, KISS1R, PROKR2, and TACR3 [24-27].

\section{Molecular Analysis of TTF1 and EAP1}

Genomic DNA was extracted from peripheral blood leukocytes with standard techniques. TTF1 is located in chromosome 14, (14q13); its three exons (NM_001079668) and putative promoter region were amplified by polymerase chain reaction (PCR) with specific exonic and intronic primer pairs. EAP1 is an intronless gene also located in chromosome 14 (14q24.3). For analysis of the EAP1 coding region (NM_0244962), five DNA fragments were amplified by PCR using exonic and boundary primers. PCR conditions and primers are available upon request. PCR products were separated on 1\% agarose gel electrophoresis, purified using EXOSAP Kit (Amersham Science, USB, Cleveland, Ohio, USA) and automatically sequenced using the ABI Prism ${ }^{\mathrm{TM}}$ Big Dye terminator Kit (Perkin-Elmer, Foster City, Calif., USA) in an ABI Prism Genetic Analyzer 3100 automatic DNA sequencer (Perkin-Elmer).

\section{EAP1 Polyalanine and Polyglutamine Tracks}

We also assessed the extent of size variation of polyglutamine (polyQ) and polyalanine (polyAla) repeats in EAP1. PCR fragments containing CAA and CAG repeats (polyglutamine track) and GCT and GCC repeats (polyalanine track) downstream of the CAG repeats were analyzed by fragment size analysis using GeneScan software (Applied Biosystems, Foster City, Calif., USA). PCR conditions and primers are available upon request.

Functional Analysis of EAP1 Polyalanine Variant

To compare the transcriptional regulatory activity of wildtype (WT) EAP1 and an EAP1 variant lacking three alanine repeats found in 2 sisters with idiopathic CPP, we used three different reporter constructs. One contained 556 bp of the KiSS1 $5^{\prime}$-flanking region (nucleotide [nt]) -529 to +27 (556 bp) [28]. Another contained a segment (nt -2345 to +399 ) of the EAP1 5 -flanking region [29], and the third consisting of a hybrid frag-

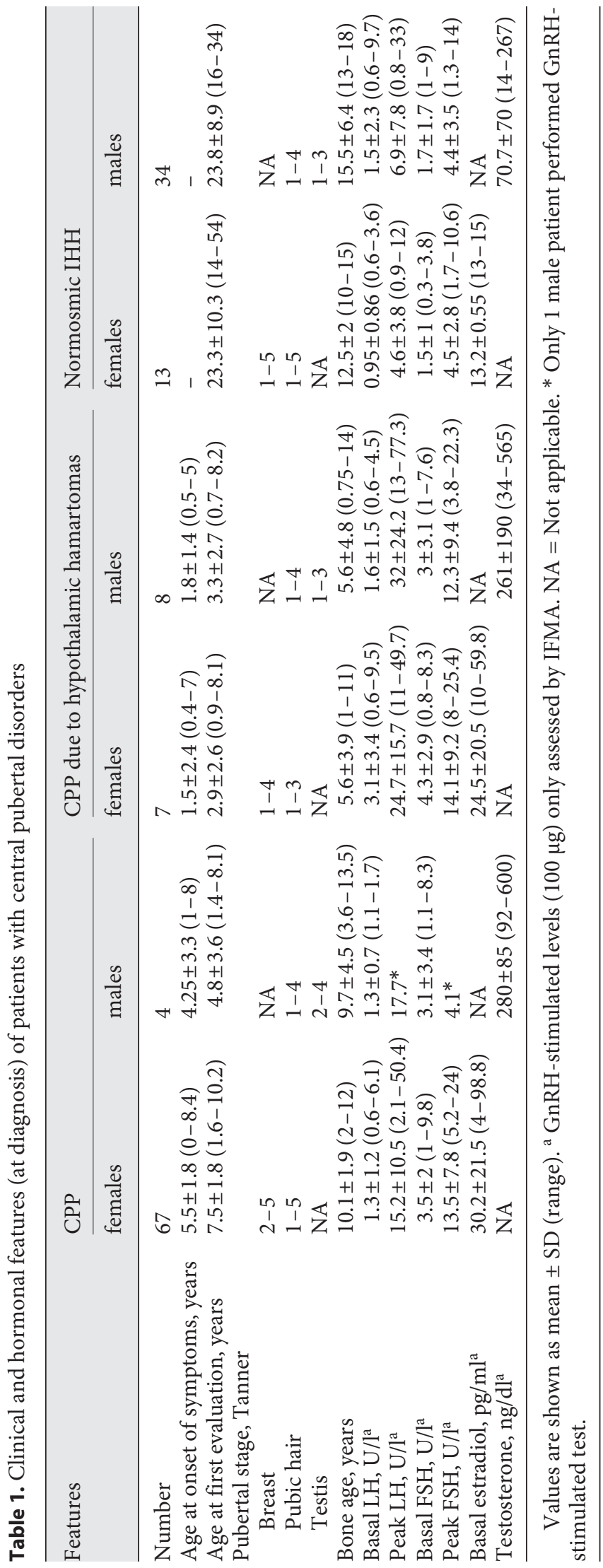

Horm Res Paediatr 2013;80:257-266 
ment of the rat Gnrh gene (NM_012767.2) containing the enhancer region $(-1869$ to -1578$)$ and proximal promoter $(-181$ to +104 ) of this gene [9]. We used this hybrid fragment for two main reasons: (a) it confers the construct the ability to respond to potential effects of EAP1 on the GnRH enhancer, and (b) its responsiveness to EAP1 is well documented [9]. All of these DNA fragments were cloned into the luciferase reporter plasmid pGL4.1 basic. A mutated EAP1 lacking 9 bp at position +1375 to +1383 (NCBI NM_024496.2) was generated utilizing site-directed mutagenesis as previously described [9]. The mutagenesis primer pair is available under request.

To compare the transcriptional regulatory activity of mutant and WT EAP1, we performed Dual Luciferase ${ }^{\circledR}$ Reporter Assays as reported previously [28]. An EAP1 mutant lacking the RING finger domain served as negative control [9]. Experiments were performed in human embryonic kidney cell line GripTite ${ }^{\mathrm{TM}} 293$ MSR cells and in the mouse GnRH neuronal cell line GT1-7.

\section{Statistical Analysis}

Quantitative variables are presented as mean \pm SD and range and qualitative variables are listed as frequencies and percentages. $\chi^{2}$ analysis or two-sided Fisher's exact test was used to compare the differences in allele and genotype distributions of the polymorphisms between CPP and IHH. For this analysis we applied the co-dominant genetic model, using the most common homozygous genotype as reference. Correction for multiple comparisons was performed using the Bonferroni adjustment ( $\mathrm{p}$ corrected $<0.006$ ). Results from the promoter assays were analyzed using a one-way analysis of variance followed by ad hoc tests (Student's t test when comparing two groups and Duncan's test when comparing several groups to a control group). Statistical significance was set at $\mathrm{p}<0.05$.

\section{Supplementary Material and Methods}

A detailed description of the Material and Methods employed to perform the TTF1 and EAP1 connectivity analysis and transcription factor binding sites prediction is provided as supplementary material (see www.karger.com/doi/10.1159/000354643).

\section{Results}

\section{Genetic Analysis of TTF1}

The entire mRNA and promoter region of TTF1 were successfully amplified and sequenced. No TTF1 allelic variants were identified in the whole cohort.

\section{Genetic Analysis of EAP1}

Amplification and sequencing of the coding region of EAP1 in all patients with central pubertal disorders revealed 4 synonymous variants, 3 of them already described in databases: p.E87E ( $r s 71428775)$, p.A163A (rs61991619) and p.Y415Y (rs879027), and a novel silent variant p.C758C (located at the RING finger region).
Table 2. Allele and genotypic frequencies of $E A P 1$ polymorphisms

\begin{tabular}{|c|c|c|c|}
\hline & $\mathrm{CPP}(\mathrm{n}=86)$ & $\mathrm{nIHH}(\mathrm{n}=47)$ & $\mathrm{p}$ \\
\hline \multicolumn{4}{|c|}{ p.E87E (rs71428775) } \\
\hline A & $132(76.7 \%)$ & $57(60.6 \%)$ & \multirow{2}{*}{0.009} \\
\hline G & $40(23.3 \%)$ & $37(39.4 \%)$ & \\
\hline AA & $56(65.1 \%)$ & $23(49 \%)$ & \multirow{2}{*}{0.1} \\
\hline GA & $20(23.2 \%)$ & $11(23.4 \%)$ & \\
\hline GG & $10(11.7 \%)$ & $13(27.6 \%)$ & 0.03 \\
\hline \multicolumn{4}{|c|}{ p.A163A (rs61991619) } \\
\hline $\mathrm{T}$ & $135(78.5 \%)$ & $77(82 \%)$ & \multirow{2}{*}{0.6} \\
\hline $\mathrm{C}$ & $37(21.5 \%)$ & $17(18 \%)$ & \\
\hline TT & $56(65.1 \%)$ & $31(66 \%)$ & \multirow{2}{*}{0.9} \\
\hline $\mathrm{CT}$ & $23(26.7 \%)$ & $15(31.9 \%)$ & \\
\hline $\mathrm{CC}$ & $7(8.2 \%)$ & $1(2.1 \%)$ & 0.3 \\
\hline \multicolumn{4}{|c|}{ p.Y415Y (rs879027) } \\
\hline $\mathrm{C}$ & $106(61.6 \%)$ & $53(56.4 \%)$ & \multirow{2}{*}{0.5} \\
\hline $\mathrm{T}$ & $66(38.4 \%)$ & $41(43.6 \%)$ & \\
\hline $\mathrm{CC}$ & $38(44.2 \%)$ & $18(38.3 \%)$ & \multirow{2}{*}{0.6} \\
\hline TC & $30(34.8 \%)$ & $17(36.1 \%)$ & \\
\hline TT & $18(21 \%)$ & $12(25.6 \%)$ & 0.7 \\
\hline \multicolumn{4}{|c|}{ p.C758C } \\
\hline $\mathrm{C}$ & $171(99.3 \%)$ & $93(98.9 \%)$ & \multirow{3}{*}{0.7} \\
\hline $\mathrm{T}$ & $1(0.7 \%)$ & $1(1.1 \%)$ & \\
\hline $\mathrm{CC}$ & $85(98.8 \%)$ & $46(97.8 \%)$ & \\
\hline CT & $1(1.2 \%)$ & $1(2.2 \%)$ & \multirow[t]{2}{*}{0.7} \\
\hline $\mathrm{TT}$ & 0 & 0 & \\
\hline
\end{tabular}

Statistical significance at $\mathrm{p}$-corrected $<0.006$.

These SNPs were identified both in patients with CPP (idiopathic and due to hypothalamic hamartoma) and $\mathrm{nIHH}$. The allele and genotypic frequencies of all EAP1 polymorphisms were not statistically different between these two groups (p-corrected $>0.006$ ) (table 2). Only the novel EAP1 variant p.C758C was also investigated in controls; the results showed that its allele and genotypic frequencies were not statistically different between controls and patients $(\mathrm{p}>0.05)$ (table 2).

In CPP patients, the length of EAP1 $5^{\prime}$-distal polyalanine region ranged from 9 to 12 alanine repeats. The most common length identified was 12 alanine residues found in 168 out of 172 alleles (97.7\%). The most common genotype was the homozygous $12 / 12$ (95.3\%). The heterozygous genotype 12/11 was identified in 2 unrelated CPP patients whereas the genotype $12 / 9$ was identified in those same 2 sisters with idiopathic CPP who had polyQ 25/22 genotype mentioned above, although their 2 first-degree female cousins with the same phenotype had the polyAla 12/12 genotype.

In the nIHH group, only the homozygous genotype containing $25 / 25$ glutamine residues was found. In the 


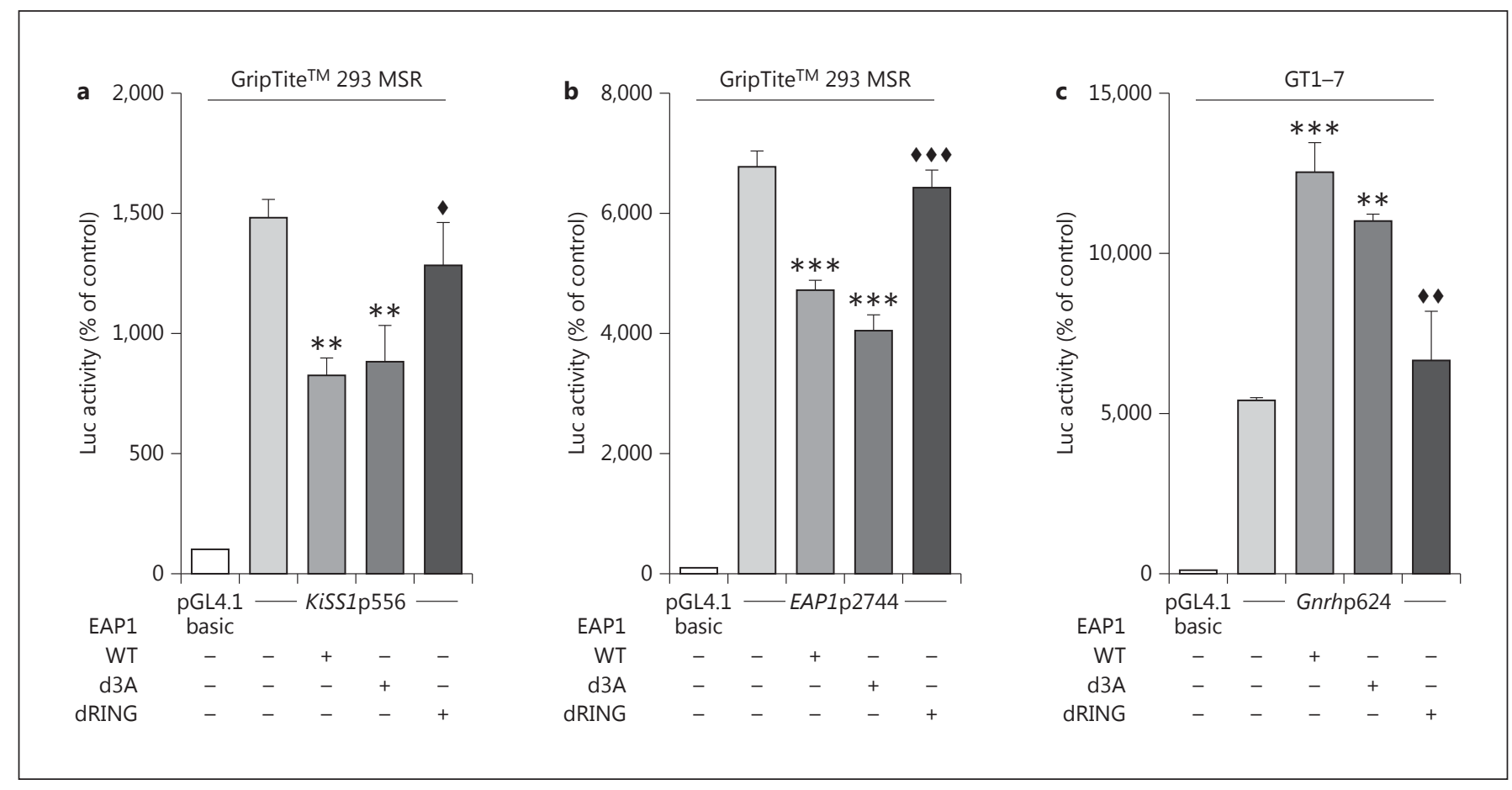

Fig. 1. Deletion of 3 alanines within the EAP1 (EAP1 d3A) coding region does not alter the repressive effect of EAP1 on (a) KiSS1 and (b) EAP1 gene expression in GripTite 293 MSR cells $(\mathrm{n}=12$ / group) or (c) the activational effect of EAP1 on Gnrh gene transcription in GT1-7 cells ( $\mathrm{n}=4$ /group). In contrast, deletion of the EAP1 RING finger domain (EAP1 dRING) abolishes both the repressive (KISS1, EAP1) and activating (Gnrh) effects of EAP1. a-c Bars are mean and vertical lines are SEM, ${ }^{*} \mathrm{p}<0.05,{ }^{* *} \mathrm{p}<0.01$, *** $\mathrm{p}<0.001$ versus promoter plasmids (KiSS1p556, EAP1p2744 or Gnrhp624) not challenged with EAP1 WT or EAP mutants, and $\bullet \mathrm{p}<0.05, " \mathrm{p}<0.01, \cdots$ groups treated with EAP1 WT. control group ( $\mathrm{n}=53,106$ alleles), the most common genotype was also the homozygous $25 / 25$, identified in 50 individuals. Two of the remaining controls had homozygous genotypes $26 / 26$ and the other control subject had the heterozygous 29/25 genotype. In the nIHH group, the length of EAP1 $5^{\prime}$-distal polyalanine region was 12 alanine residues in all alleles. Finally, in the control group ( $n=53,106$ alleles), the most common genotype was also the homozygous $12 / 12$, identified in 51 individuals. The remaining 2 controls had heterozygous genotypes (12/11 and 12/16 alanine residues). There was no significant allele and genotype difference of length of alanine tract among the groups $(\mathrm{p}>0.05)$.

Because 2 sisters with CPP showed the EAP1 phenotype $12 / 9$, we explored the possibility that this shorter EAP1 $5^{\prime}$-distal polyalanine region could have functional consequences. Promoter assays using the KISS1, Gnrh, and $E A P 1$ promoters revealed that this $E A P 1$ variant behave like WT EAP1, inhibiting KISS1 and EAP1 transcriptional activity and activating the Gnrh promoter (fig. 1).

\section{Network Node Degree Analysis of EAP1 and TTF1}

Connectivity to Genes Influencing the Age of Menarche

Although neither EAP1 nor TTF1 variants are associated to alterations in the timing of puberty, the possibility exists that variants in regulatory regions of genes targeted by EAP1 and/or TTF1 may affect the ability of EAP1 and/or TTF1 to modulate expression of these target genes, and thus affect the timing of puberty. As a first step towards assessing this possibility, we performed a network analysis to determine if EAP1, TTF1 or both are functionally linked to genes implicated in controlling the timing of menarche. We examined the EAP1 and TTF1 connectivity to 44 genes implicated by genome-wide association studies (GWAS) as influencing the time of menarche [30-33]. We observed dense interconnectivity among the different menarche-associated genes, including KISS1 and KISS1R, and connectivity of these genes with EAP1 (online suppl. fig. 1). A similar analysis of TTF1 connectivity, including information about transcription factor binding sites predicted by the MATCH 


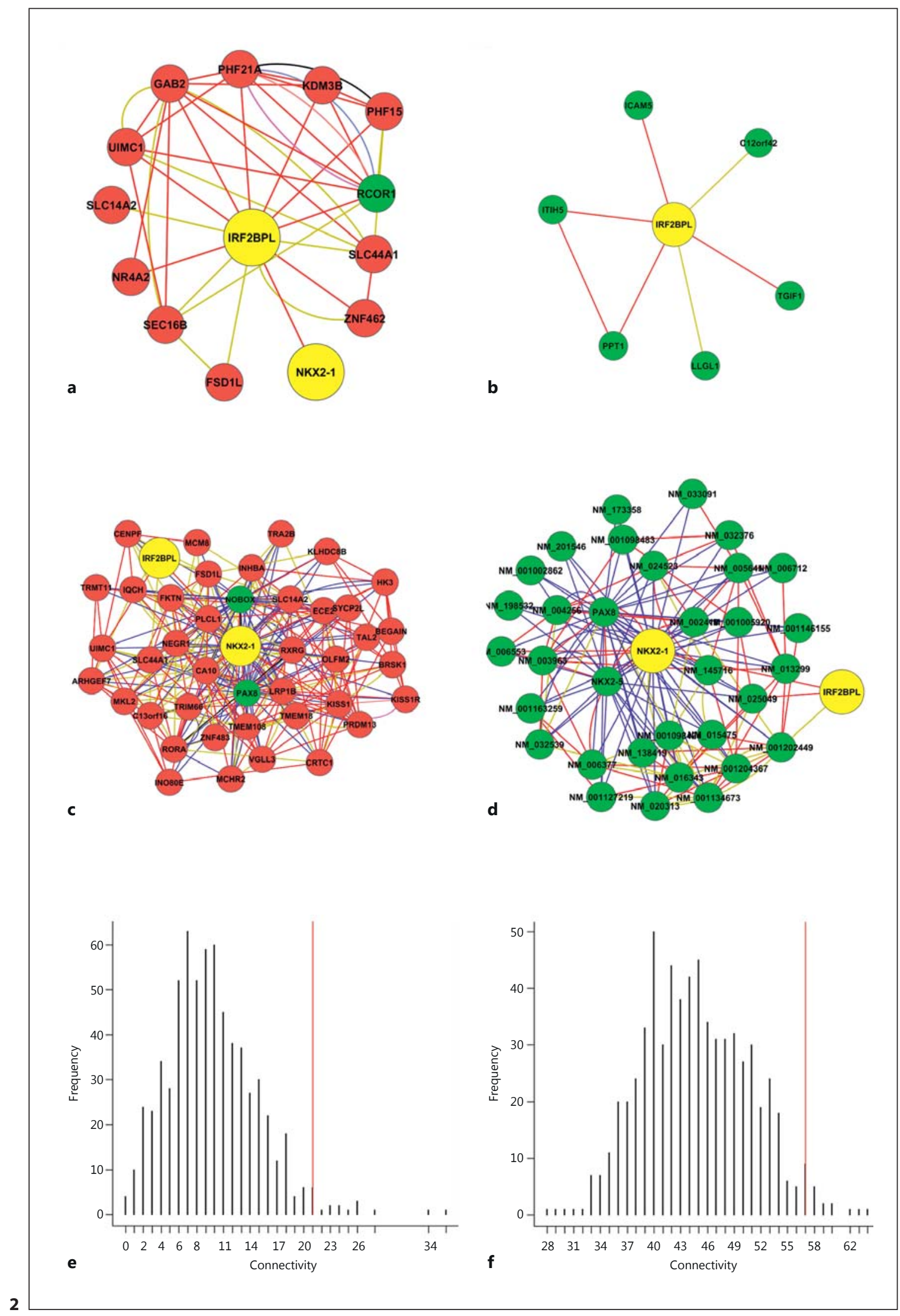


software (online suppl. information), yielded a network with similar characteristics (online suppl. fig. 2).

We then constructed subnetworks to visualize the genes more densely connected to either EAP1 or TTF1 (termed first neighbors) and any edges (connections) that exist between those first neighbors. We performed the same analysis on an ensemble of 1,000 networks of the same size containing randomly selected gene sets unrelated to menarche from the GeneMANIA database [34]. Utilizing this approach, we found that the node degree of EAP1 with its menarche-associated gene subnetwork was higher $(\mathrm{p}=0.037)$ than in a majority (642 of 666) of successfully generated randomly selected subnetworks at a single-sided $a$ of 0.05 (fig. 2a, b). The first neighbors are themselves highly connected to one another, with a mean node degree of 5.38. In contrast, the EAP1 first-neighbor networks generated from randomly selected genes is sparse and poorly interconnected (fig. 2b), with a mean node degree of only 2. TTF1's node degree within the menarche-associated gene subnetwork is also greater than its expected node degree within the randomly generated subnetworks (646 of 667 networks, $\mathrm{p}=0.03$ ). Though

Fig. 2. Connectivity of EAP1 and TTF1 to genes from a menarcheassociated gene set predicted to function as first neighbors of a subnetwork depicting EAP1 and TTF1 as central hubs. a EAP1 connectivity to menarche-associated genes operating as first neighbors. b Lack of EAP1 connectivity to a set of randomly selected genes. c TTF1 connectivity to menarche-associated genes operating as first neighbors. d Reduced TTF1 connectivity to a set of randomly selected genes. The EAP1 subnetwork is derived from the GeneMANIA database only; the TTF1 subnetwork derives from both GeneMANIA data and from the detection of protein-DNA interactions by TRANSFAC. Edges (connections) are color-coded by interaction type; redundant edges of the same type from different data sources are not shown. Red circles are menarches-associated genes; green circles represent additional genes predicted by GeneMANIA to be part of the subnetwork. The EAP1 node is also connected to the TTF1 node (yellow circles). e Plot of connectivity distribution of EAP1 in first-neighbor subnetworks of randomly selected gene sets generated by the GeneMANIA database. The number of EAP1 connections detected in the menarche-associated gene first-neighbor subnetwork is indicated by the red line, visually demonstrating the significant increase in connections of EAP1 to menarche-associated genes relative to the connectivity expected from random gene sets. f Plot of connectivity distribution of TTF1 in first-neighbor subnetworks of randomly selected gene sets generated by the GeneMANIA database and including TTF1 predicted binding sites. The number of TTF 1 connections detected in the menarche-associated gene firstneighbor subnetwork is indicated by the red line. Red = Co-expression; grey = shared protein domain; green = genetic interaction; orange = predicted interaction; purple = shared pathway membership; blue $=$ other (including predicted TFBS binding).

TTF-1 and EAP1 in Central Pubertal Disorders less visually striking (fig. $2 \mathrm{c}, \mathrm{d}$ ), the difference in node degree among first neighbors in the menarche-associated versus random subnetworks was clearly discernible (mean node degrees of 13.81 vs. 9.2, respectively).

Finally, visualization of the first-neighbor subnetworks of EAP1 and TTF1 demonstrated that the number of connections between the members of their respective menarche-associated subnetworks was strikingly greater than the connectivity observed between the genes in random gene set-derived subnetworks (fig. 2e, f).

Interestingly, three genes encoding transcriptional repressors are predicted to be first neighbors of the EAP1 or TTF1 network or both. Two of these genes belong to the large zinc finger (ZNF) gene family of transcriptional repressors [35] (ZNF462 and ZNF483, respectively). The other is $F S D 1 L$, a gene encoding a protein that interacts with HDAC2 [36], a histone deacetylase. Both KISS1 and KISS1R are predicted to be first neighbors of TTF1, and both are detected as secondary nodes within the expanded EAP1 network (online suppl. fig. 1). Unexpectedly, several genes encoding solute carrier proteins expressed in brain [3739], were predicted to be first neighbors in both networks. While SLC14A2, a gene encoding a urea transporter [37], is a first neighbor for both the EAP1 and TTF1 network, the related gene SLC14A1 is predicted to be only an EAP1 first neighbor. Conversely, SLC44A1, which encodes a choline transporter [38, 39], is a TTF1 first neighbor.

\section{Discussion}

In the present study, we examined a cohort of $86 \mathrm{sub-}$ jects with CPP and 47 subjects with nIHH to determine if these disorders affecting the time of puberty are associated with mutations in TTF1 and EAP1, two genes encoding transcriptional regulators previously implicated in the control of puberty in experimental animals $[9,10]$. Our interest in these two genes was prompted by several observations: (a) conditional deletion of the Ttf1 gene from brain neurons or RNAi-mediated silencing of the Eap1 gene targeted to the rodent hypothalamus results in delayed puberty, diminished reproductive capacity and disrupted estrous cyclicity $[9,10]$; (b) silencing the EAP1 gene in the hypothalamus of non-human primates abolishes menstrual cyclicity [11], and (c) hypothalamic expression of both Ttf1 and Eap1 increases immediately before or at the time of puberty in a gonad-independent manner $[8,40]$. These observations notwithstanding, the present results demonstrate that neither gene had functional mutations in subjects with CPP, CPPH or nIHH.

Horm Res Paediatr 2013;80:257-266 
Inactivating mutations of the TTF1 gene are associated with neurological disorders, hypothyroidism and pulmonary defects $[41,42]$. Despite this phenotypic heterogeneity, the existence of reproductive abnormalities in these patients has not been reported. None of our patients with pubertal disorders exhibited signs of neurological, pulmonary or thyroid dysfunction, a finding consistent with the absence of mutations or polymorphisms in these genes. Our results do not exclude the possibility that mutations may be found in much larger cohort of patients, or that sequence variation in upstream $5^{\prime}$-flanking regulatory region of the $T T F 1$ gene may result in either a more pronounced response to transcriptional activators or greater repression by transcriptional silencers.

Thus far, inactivating mutations of the EAP1 gene in humans have not been reported and an Eap1 null mouse is not yet available. Although the EAP1 gene appeared functionally intact in our patients, it is formally possible that inactivating mutations may be found upon analysis of much larger populations of affected individuals or that single nucleotide polymorphisms affecting either the transcriptional regulation of EAP1 or the stability of its encoded mRNA may be discovered. A precedent for this potential outcome has been provided by the recent demonstration that a single-nucleotide polymorphism in the $5^{\prime}$-flanking region of the EAP1 gene is associated with increased incidence of amenorrhea/oligomenorrhea in rhesus monkeys [43].

Though still limited, our knowledge of the genetic mechanisms involved in the neuroendocrine control of human puberty is steadily increasing. Studies from several laboratories have made clear that no isolated pathway or cellular subset is responsible for the onset of puberty [1, 44-46]. In fact, if all genes implicated in the genesis of IHH, including those involved in $\mathrm{GnRH}$ neuronal migration (KAL-1, FGFR1, FGF8, NELF, PROK2, PROKR2, TAC3 and TAC3R), GnRH action (GnRHR, KiSS1, KISS1R) and gonadotropin action $(L H \beta, F S H \beta)$ are considered together, it becomes evident that they contribute to only $30 \%$ of $\mathrm{IHH}$ cases [27]. Therefore, the molecular basis of $\mathrm{IHH}$, and in particular nIHH, remains to be established in most patients with this disorder. Much less is known about the genetic component of CPP, although there is evidence that at least $25 \%$ of such cases have a familial origin [14]. Within this group, a KISS1R-activating mutation in a girl with CPP and a KiSS1-activating mutation in a boy with CPP have been reported $[15,16]$. Recently, using whole-exome sequencing in 40 members of 15 families with CPP, Abreu et al. [47] identified mutations in the imprinted gene MKRN3 in 5 families, describing a new genetic cause of familial $\mathrm{CPP}$. Thus several other genes may contribute to the pu- bertal process. Consistent with this idea, GWAS have demonstrated that sequence variations in more than 30 genes are associated with an early age at menarche [30-33, 48].

These observations have led to the concept that pubertal process is controlled by highly coordinated and interactive gene networks organized in a hierarchical fashion $[1,7]$. According to this concept, the core of these networks is composed of genes encoding transcriptional regulators that direct expression of downstream subordinate genes expressed within both neuronal and glial subsets involved in initiating the pubertal process. TTF1 and EAP1 are thought to be two of such genes.

The lack of association between TTF1 and EAP1 sequence variation and disorders of puberty suggests that these genes do not play a role in the regulation of human puberty. However, before reaching this conclusion it is important to consider an important feature of polygenic diseases. De novo mutations in autisms spectrum disorder (ASD), a well-recognized polygenic disorder, do not display a rate of mutation that can be connected to the phenotypic manifestations of ASD [21]. Instead, these genes display a high degree of connectivity, not only among themselves but also to other genes previously implicated in ASD, leading to the conclusion that 'de novo events that confer risk of ASD are distributed across many genes and are incompletely penetrant' [21].

Applying a similar approach, but using different tools, we now show that both EAP1 and TTF1 are functionally connected to a set of genes shown by GWAS to influence the age at menarche [30-33, 48]. We observed that these menarche-associated genes form densely interconnected networks with both EAP1 and TTF1. An in-depth analysis of the subnetworks formed by the genes most connected to EAP1 and TTF1 revealed that these 'first neighbors' include at least three transcriptional repressors. ZNF462 and ZNF483, two members of the ZNF gene family [35], and FSD1L (fibronectin type III and SPRY domain-containing 1-like peptide), a gene encoding a protein that interacts with HDAC2 [36], a histone deacetylating enzyme involved in repressing gene transcription [49]. In addition to these repressors, the TTF1 network has KISS1 and KISS1R as first neighbors, a finding consistent with the observation that kisspeptin neurons express TTF1 mRNA and KISS1 transcription is trans-activated by TTF1 [10]. Neither KISS1 nor KISS1R are first neighbors of EAP1, but they are predicted to be secondary nodes in the expanded EAP1 network that contains all other menarche-associated genes. An unexpected finding was the detection of genes encoding solute carrier proteins (SLC14A1, SLC44A1 and SLC14A2) as first 
neighbors in both networks. Although the biological significance of these findings remains to be established, sequence variation near these genes is associated with the timing of puberty, and all of them are expressed in brain, including the hypothalamus (http://www.brainmap.org/) [37-39]. Also of interest is the relationship of SLC44A1 to cholinergic transmission, as SLC44A1 encodes a choline transporter involved in sustaining the synthesis of acetycholine [38, 39]. Further work will be necessary to elucidate the role that these genes may play in influencing the timing of human puberty.

The finding that EAP1 and TTF1 are strongly connected to menarche-associated genes raises the intriguing possibility that sequence variation affecting relevant distal and/or proximal regulatory regions may alter the ability of TTF1/EAP1 to interact with such regions and regulate the transcriptional activity of these genes. The same holds true for sequence variation affecting the EAP1 gene itself. Three observations provide initial support for this concept: one is the finding that a SNP in the $5^{\prime}$-flanking region of the $E A P 1$ is associated with hypothalamic amenorrhea in nonhuman primates and with the loss of a SMAD3 site required for TGF $\beta$-dependent activation of EAP1 transcription [43]. The second observation is the presence of a SNP (rs10980926) overlapping a predicted TTF1 site in an intron of ZNF483; this SNP was identified as associated with an earlier age at menarche in a meta-analysis of GWAS studies [46]. While the variation itself is common and does not adversely affect the TTF1 site, the SNP also overlaps a 4-bp deletion polymorphism (rs200459103). If this deletion occurs, the core of the predicted TTF1 site is removed and replaced with a pair of retinoic acid response motifs (RXR- $\alpha / P P A R-~ \gamma$ and VDR/CAR/PPR). The third observation comes from the present study: the novel EAP1 variant we are reporting and the nearby rs 879027 SNP overlap ChIP-seq peaks identified by the ENCODE project [48] for the factors SMARCB1 and c-Myc, respectively, despite being located in the gene body of EAP1. It is therefore plausible that these variants could affect the binding activity of these or other related TFs, like the 5 '-flanking variant does. While the presence of a set of variants alone is not sufficient to explain abnormalities in the timing of puberty, it does suggest contribution to a polygenic effect, in which several variants would operate in a combinatorial fashion to alter pubertal timing through cumulative effects on the transcriptional control network involved in the initiation and control of puberty. The validity of this notion needs to be assessed by first identifying menarche-associated sequence variations affecting TTF1/EAP1 interaction with these genomic regions, as well as the effect of EAP1 sequence vari- ation on the binding of transcription factors, followed by studies of the resulting biological outcome.

In conclusion, our results show the lack of germline or de novo variants of the TTF1 gene and its promoter in patients with either idiopathic central pubertal disorders (CPP and $\mathrm{nIHH}$ ) or CPP due to hypothalamic hamartoma (CPPH). We also report that in contrast to TTF1, which is a highly conserved gene, EAP1 is a polymorphic gene. Its variants, however, are not associated with central pubertal disorders in the cohorts we analyzed. Nevertheless, our study also provides evidence for the concept that sequence variation in individual genes implicated in the control of puberty, such as EAP1 and TTF1, may not by itself confer susceptibility to disease, unless there are simultaneous alterations in other genes functionally connected via the architectural hierarchy of a gene network. Because binding sites for transcriptional regulators are not restricted to promoter regions, transcription factors might also regulate gene expression by acting at distal sites, or sites in the gene body downstream from the promoter region. This feature may be especially meaningful if distal regions containing transcription factor binding motifs also function as enhancer. Should this be the case, sequence variation affecting these sites may compromise the ability of a transcription factor to regulate expression via binding to that enhancer region, and therefore, affect the overall output of the network.

\section{Acknowledgments}

This work was supported by FAPESP (06/56531-0 to P.C.) and CNPq (305743/2011-2 to B.B.M., 300982/2009-7 to I.J.P.A. and 302825/2011-8 to A.C.L.), the US National Science Foundation (NSF IOS1121691 to S.R.O.), NIH grant 8P51-OD-011092-53 for the operation of the Oregon National Primate Research Center (S.R.O.), and the German Research Foundation Grant HE 3151/41 (S.H.). H.W. was a postdoctoral fellow supported by NIH Training Grant T32 HD007133.

\section{Disclosure Statement}

The authors have no conflicts of interest to disclose.

References 
3 Plant TM: Hypothalamic control of the pituitary-gonadal axis in higher primates: key advances over the last two decades. J Neuroendocrinol 2008;20:719-726.

4 Kimura S, Hara Y, Pineau T, et al: The T/ebp null mouse: thyroid-specific enhancer-binding protein is essential for the organogenesis of the thyroid, lung, ventral forebrain, and pituitary. Genes Dev 1996;10:60-69.

$\checkmark 5$ De Felice M, Di Lauro R: Thyroid development and its disorders: genetics and molecular mechanisms. Endocr Rev 2004;25:722-746.

6 Rampazzo A, Pivotto F, Occhi G, et al: Characterization of C14orf4, a novel intronless human gene containing a polyglutamine repeat, mapped to the ARVD1 critical region. Biochem Biophys Res Commun 2000;278:766774.

7 Ojeda SR, Dubay C, Lomniczi A, et al: Gene networks and the neuroendocrine regulation of puberty. Mol Cell Endocrinol 2010;324:311.

8 Lee BJ, Cho GJ, Norgren RB Jr, et al: TTF-1, a homeodomain gene required for diencephalic morphogenesis, is postnatally expressed in the neuroendocrine brain in a developmentally regulated and cell-specific fashion. Mol Cell Neurosci 2001;17:107-126.

-9 Heger S, Mastronardi C, Dissen GA, et al: Enhanced at puberty 1 (EAP1) is a new transcriptional regulator of the female neuroendocrine reproductive axis. J Clin Invest 2007; 117:2145-2154.

10 Mastronardi C, Smiley GG, Raber J, et al: Deletion of the Ttf1 gene in differentiated neurons disrupts female reproduction without impairing basal ganglia function. J Neurosci 2006;26:13167-13179.

$\checkmark 11$ Dissen GA, Lomniczi A, Heger S, Neff TL, Ojeda SR: Hypothalamic EAP1 (enhanced at puberty 1 ) is required for menstrual cyclicity in non-human primates. Endocrinology 2012;153:350-361.

-12 Palmert MR, Boepple PA: Variation in the timing of puberty: clinical spectrum and genetic investigation. J Clin Endocrinol Metab 2001;86:2364-2368.

13 Pitteloud N, Durrani S, Raivio T, Sykiotis GP: Complex genetics in idiopathic hypogonadotropic hypogonadism. Front Horm Res 2010; 39:142-153.

14 De Vries L, Kauschansky A, Shohat M, Phillip M: Familial central precocious puberty suggests autosomal dominant inheritance. J Clin Endocrinol Metab 2004;89:1794-1800.

-15 Teles MG, Bianco SD, Brito VN, et al: A GPR54-activating mutation in a patient with central precocious puberty. $\mathrm{N}$ Engl J Med 2008;358:709-715.

16 Silveira LG, Noel SD, Silveira-Neto AP, et al: Mutations of the KISS1 gene in disorders of puberty. J Clin Endocrinol Metab 2010;95: 2276-2280.

17 Ko JM, Lee HS, Hwang JS: KISS1 gene analysis in Korean girls with central precocious puberty: a polymorphism, p.P110T, suggested to exert a protective effect. Endocr J 2010;57:701-709.
18 Arita K, Kurisu K, Kiura Y, Iida K, Otsubo H: Hypothalamic hamartoma. Neurol Med Chir (Tokyo) 2005;45:221-231.

19 Carel JC, Leger J: Clinical practice. Precocious puberty. N Engl J Med 2008;358:2366-2377.

20 Brito VN, Latronico AC, Arnhold IJ, Mendonca BB: Update on the etiology, diagnosis and therapeutic management of sexual precocity. Arq Bras Endocrinol Metabol 2008;52: $18-31$.

21 Neale BM, Kou Y, Liu L, et al: Patterns and rates of exonic de novo mutations in autism spectrum disorders. Nature 2012;485:242245.

22 George JT, Seminara SB: Kisspeptin and the hypothalamic control of reproduction: lessons from the human. Endocrinology 2012; 153:5130-5136

23 Davidson TM, Murphy C: Rapid clinical evaluation of anosmia. The alcohol sniff test. Arch Otolaryngol Head Neck Surg 1997;123:591594.

24 Abreu AP, Trarbach EB, de Castro M, et al: Loss-of-function mutations in the genes encoding prokineticin-2 or prokineticin receptor-2 cause autosomal recessive Kallmann syndrome. J Clin Endocrinol Metab 2008;93: 4113-4118.

25 Trarbach EB, Costa EM, Versiani B, et al: Novel fibroblast growth factor receptor-1 mutations in patients with congenital hypogonadotropic hypogonadism with and without anosmia. J Clin Endocrinol Metab 2006; 91:4006-4012.

26 Teles MG, Trarbach EB, Noel SD, et al: A novel homozygous splice acceptor site mutation of KISS1R in two siblings with normosmic isolated hypogonadotropic hypogonadism. Eur J Endocrinol 2010;163:29-34.

27 Beate K, Joseph N, Nicolas de R, Wolfram K: Genetics of isolated hypogonadotropic hypogonadism: role of $\mathrm{GnRH}$ receptor and other genes. Int J Endocrinol 2012;2012:147893

28 Mueller JK, Dietzel A, Lomniczi A, et al: Transcriptional regulation of the human KiSS1 gene. Mol Cell Endocrinol 2011;342:8-19.

29 Mueller JK, Koch I, Lomniczi A, et al: Transcription of the human EAP1 gene is regulated by upstream components of a pubertycontrolling tumor suppressor gene network. Mol Cell Endocrinol 2012;351:184-198.

30 Ong KK, Elks CE, Li S, et al: Genetic variation in LIN28B is associated with the timing of puberty. Nat Genet 2009;41:729-733.

31 Perry JR, Stolk L, Franceschini N, et al: Metaanalysis of genome-wide association data identifies two loci influencing age at menarche. Nat Genet 2009;41:648-650.

- 32 Sulem P, Gudbjartsson DF, Rafnar T, et al: Genome-wide association study identifies sequence variants on 6q21 associated with age at menarche. Nat Genet 2009;41:734-738.

-33 He C, Kraft P, Chen C, et al: Genome-wide association studies identify loci associated with age at menarche and age at natural menopause. Nat Genet 2009;41:724-728.
34 Warde-Farley D, Donaldson SL, Comes O, et al: The GeneMANIA prediction server: biological network integration for gene prioritization and predicting gene function. Nucleic Acids Res 2010;38:W214-W220.

35 Urrutia R: KRAB-containing zinc-finger repressor proteins. Genome Biol 2003;4:231.

36 Lin YY, Kiihl S, Suhail Y, et al: Functional dissection of lysine deacetylases reveals that HDAC1 and p300 regulate AMPK. Nature 2012;482:251-255.

37 Fenton RA, Stewart GS, Carpenter B, et al: Characterization of mouse urea transporters UT-A1 and UT-A2. Am J Physiol Renal Physiol 2002;283:F817-F825.

38 Yamada T, Inazu M, Tajima H, Matsumiya T: Functional expression of choline transporterlike protein 1 (CTL1) in human neuroblastoma cells and its link to acetylcholine synthesis. Neurochem Int 2011;58:354-365.

-39 Machova E, O'Regan S, Newcombe J, et al: Detection of choline transporter-like 1 protein CTL1 in neuroblastoma $\mathrm{x}$ glioma cells and in the CNS, and its role in choline uptake. J Neurochem 2009;110:1297-1309.

40 Matagne V, Mastronardi C, Shapiro RA, Dorsa DM, Ojeda SR: Hypothalamic expression of Eap1 is not directly controlled by ovarian steroids. Endocrinology 2009; 150:18701878.

41 Breedveld GJ, van Dongen JW, Danesino C, et al: Mutations in TITF-1 are associated with benign hereditary chorea. Hum Mol Genet 2002;11:971-979.

42 Krude H, Schutz B, Biebermann H, et al: Choreoathetosis, hypothyroidism, and pulmonary alterations due to human NKX2-1 haploinsufficiency. J Clin Invest 2002;109:475-480.

-43 Lomniczi A, Garcia-Rudaz C, Ramakrishnan $\mathrm{R}$, et al: A single-nucleotide polymorphism in the EAP1 gene is associated with amenorrhea/ oligomenorrhea in non-human primates. Endocrinology 2012;153:339-349.

44 Krewson TD, Supelak PJ, Hill AE, et al: Chromosomes 6 and 13 harbor genes that regulate pubertal timing in mouse chromosome substitution strains. Endocrinology 2004; 145: 4447-4451.

45 Eaves L, Silberg J, Foley D, et al: Genetic and environmental influences on the relative timing of pubertal change. Twin Res 2004;7:471481

46 Gajdos ZK, Butler JL, Henderson KD, et al: Association studies of common variants in 10 hypogonadotropic hypogonadism genes with age at menarche. J Clin Endocrinol Metab 2008;93:4290-4298.

47 Abreu AP, Dauber A, Macedo DB, et al: Central precocious puberty caused by mutations in the imprinted gene MKRN3. N Engl J Med 2013;368:2467-2475

48 Elks CE, Perry JR, Sulem P, et al: Thirty new loci for age at menarche identified by a metaanalysis of genome-wide association studies. Nat Genet 2010;42:1077-1085.

49 Kouzarides T: Chromatin modifications and their function. Cell 2007;128:693-705. 